\title{
Complete Green Synthesis of Gold Nanoparticles using Laser Ablation in Deionized Water Containing Chitosan and Starch
}

\section{Tran X. Phuoc*}

National Energy Technology Laboratory, Department of Energy, Pittsburgh, United States

*Corresponding author: Tran X. Phuoc, National Energy Technology Laboratory, Department of Energy, P. O. Box 10940, MS 84-340, Pittsburgh, PA 15261, E-mail: tran@netl.doe.gov

Citation: Tran X. Phuoc (2014) Complete Green Synthesis of Gold Nanoparticles using Laser Ablation in Deionized Water Containing Chitosan and Starch. J Mater Sci Nanotechnol 2(2): 202. doi: 10.15744/23489812.1.401

Received Date: February 27, 2014 Accepted Date: December 02, 2014 Published Date: December 09, 2014

\begin{abstract}
In this paper we report our results on the green synthesis of nontoxic, stable, and small size gold nanoparticle suspensions. We used the single and two-beam laser to ablate a gold target submerged in deionized water containing chitosan or starch as the stabilizing agent. Since both chitosan and starch are biodegradable and biocompatible, use of these natural polymers for gold nanoparticle protection and stabilization does not introduce any environmental toxicity or biological hazards. Our results show that ablating in neat deionized water, the ablated particles were not stable, they agglomerated and connected together in random structures. When gold was ablated in $\mathrm{dw}$ containing either chitosan or starch, the resulting suspension became stable with smaller, isolated, and well dispersed particles. The particles ablated by the single-beam ablation approach were much larger in size and side distribution compared with those produced using the two-beam ablation approach.
\end{abstract}

Keywords: Single-beam laser ablation; Two-beam laser ablation; Chitosan; Starch; Gold nanoparticles

\section{Introduction}

Gold nanoparticles have been found as a valuable material in many different fields, from material to medical sciences. Gold nanoparticles have been used for single electron devices. $\mathrm{TiO}_{2}$-supported gold nanoparticles display a highly selective catalytic activity for $\mathrm{CO}$ oxidation at low temperature. For medical applications, gold nanoparticles can be used as ultrasensible biosensors, cancer identifiers, thermal treatments of localized prostate, kidney and many other cancer cells. For these applications, the particles should be stable, pure, well-controlled size, shape and narrow size distribution.

Gold nanoparticles can be synthesized chemically or physically. In the chemical approach, gold nanoparticles have been synthesized by the reduction of a diluted metal salt in an aqueous solutions composed of a reducing reagent and a stabilizing surfactant such as cetylrimethylammonium bromide (CTAB). Such a chemical approach can easily yield stable gold nanoparticles or nanorods with plasmon absorption peaks ranging from visible to NIR wavelengths. However, Au nanoparticles/nanorods produced by this technique are not free from undesired toxic components whose micelle concentrations might be much higher than the reported threshold for cytotoxicity for collaterally damaging healthy cells or tissues [1]. In the physical approach, the laser ablation in liquid technique has been used. With this technique, a high-power laser beam is focused for an appropriate time onto a gold target that is submerged in a liquid environment. The solid and the liquid layer immediately adjacent to the solid surface are heated, melted and ionized rapidly producing a high temperature, high pressure plasma plume containing highly ionized species. Subsequently, these excited species are ejected and react with each other and with the liquid molecules to form nanoparticles that are supercooled by the surrounding liquid. The technique has been used successfully to generate nanoparticles and nanomaterials by ablating metals and metal oxides in deionized water and solvents [2-12]. In terms of the final product purity, this technique is more advantageous than the chemical one because it can generate stable nanoparticles without the need of using chemical reduction reactions or ion exchange, thus, nanoparticles obtained are free of extraneous ions or other chemicals. Additional benefits can be deduced from the influence of the liquid environment which can provide further chemical effects such as oxidation, surface functionalization, etc. and physical effects such as cooling and confinement for size and size distribution control. The disadvantage is, however, that the particle size distributions of the nanoparticles produced by this technique tend to be widened. This is possibly due to agglomeration and ejection of the relatively large metal fragments during the ablation process. To obtained narrow particle size distribution, different surfactants must be used. Thus, the problem of chemical contamination cannot be eliminated.

In this paper we report our results on the synthesis of gold nanoparticles using the laser ablation in liquid technique and we use chitosan or starch as the stabilizing agent. The ablation uses two lasers that are arranged in a cross-beam configuration. One beam, called ablating beam, is focused on the gold target and one beam is focused slightly away from the target surface and onto the vapor plume generated by the ablating beam. This arrangement is better than the conventional single-beam laser ablation because the ablated particles by the ablating beam can be effectively further ablated into smaller size and pushed away from the solid surface by the second beam allowing the ablating beam to reach the solid surface without being significantly absorbed. Thus, the technique not only generates small size particles but also yields higher ablation rate. 
Chitosan is a cationic surfactant. It is one of the most important natural polymers isolable from crustacean cell. Being a cationic polysaccharide, chitosan has a high positive charge that counteracts the anionic charge of the gold nanoparticles once they are dispersed within the solution. Starch, on the other hand, can be considered as an anionic surfactant. It is a renewable polymer formed by the $\alpha-1,4$ linkages between D-glucose units and adopts a left-handed helical conformation in aqueous solution. When dissolved in water, starch can facilitate the complexation of metal ions to the molecular matrix by its extensive number of hydroxyl groups. With their excellent biodegradable and biocompatible characterisrics, using chitosan or starch for protection and stabization of gold nanoparticles will not introduce any environmental toxicity or biological hazards. Although these natural polymers have been used as the effective reducing and stabilizing agents in the chemical synthesis of silver, gold nanoparticles [13-22], use of these natural polymers to stabilize and control the morphology of the nanoparticles produced by the laser ablation in liquid technique has not been investigated. We study this problem because there are several key advantages to the use of these renewable polymers as the protecting agents. First it is possible to form a dispersion of chitosan or starch in $\mathrm{H}_{2} \mathrm{O}$, and so one can completely avoid the use of organic solvents. Second, the binding interaction between starch and the metal molecules is relatively weak as compared to the interactions between the nanoparticles and typical thiol-based protecting groups. This implies that the protection should be easily reversible at relatively higher temperatures. Finally, due to its water solubility, no toxicity, biocompatibility, biodegradability, and gel/film-forming properties, chitosan or starch-protected nanoparticles can be readily integrated into systems relevant for pharmaceuticals and biomedical applications.

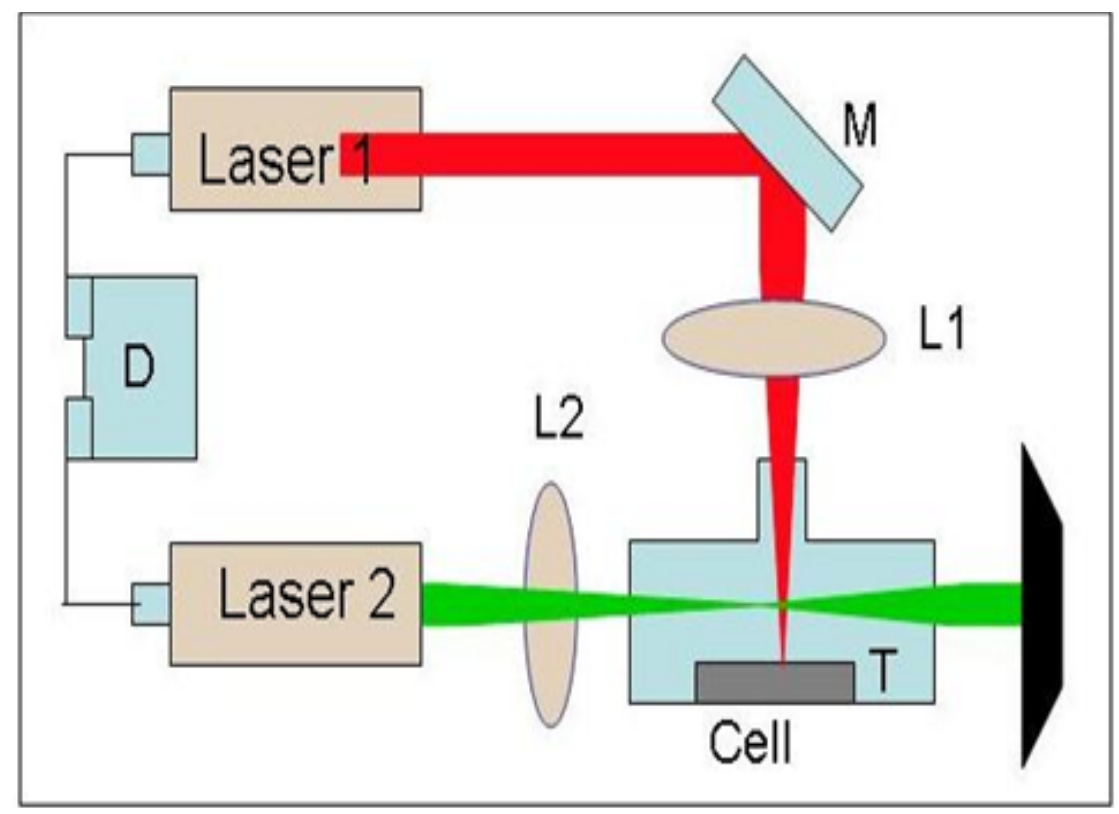

Figure 1: A sketch of the present laser ablation in liquid apparatus.

\section{Experiments}

The present experimental apparatus was described in details elsewhere [7] and it will not be repeated here. In summary, the laser ablation apparatus used two single-mode, Q-switched Nd-Yag lasers to create two laser beams that were arranged in a cross beam configuration as shown in Figure 1. In this study, the lasers were operated at $10 \mathrm{~Hz}$ rep rate with a pulse duration of $5.5 \mathrm{~ns}$. The laser beams were $10 \mathrm{~mm}$ below the liquid surface and the metal target was located at $50 \mathrm{~mm}$ from the cell window facing the ablation beam. The fluence of the ablation beam was $0.265 \mathrm{~J} / \mathrm{cm}^{2}$ and the wavelength was $1064 \mathrm{~nm}$. The wavelength and the fluence of the second beam were constant at $0.09 \mathrm{~J} / \mathrm{cm}^{2}$ and $532 \mathrm{~nm}$, respectively. Gold sample ( 25 x $25 \mathrm{~mm}$ and $1 \mathrm{~mm}$ thick) with $99.998 \%$ purity was purchased from Alfa Aesar. The sample had a smooth surface and it was used as received. Chitosan ( $>75 \%$ 80\% deacetylation) and starch were purchased from Sigma-Aldrich. Medium molecular weight (MW) chitosan had a molecular weight of $103.200 \mathrm{~g} /$ mol and low molecular weight chitosan had a molecular weight of $72.189 \mathrm{~g} / \mathrm{mol}$.

When chitosan was used, the liquid base was prepared by mixing 0.1 weight percent (wt\%) chitosan into deionized water (dw) having 0.5 vol\% acetic acid then stirred for 24 hours using a magnetic stirrer. When starch (corn or potato) was used, the liquid base was prepared by mixing $1 \mathrm{wt} \%$ starch with neat deionized water for 24 hours and the remaining starch particles were removed by centrifuging the solution at 20,000 rpm for 45 minutes. The concentrations of chitosan and starch used here are found to be in the levels that effectively stabilize the ablated nanoparticles.

Colloidal suspensions were obtained and carefully sampled for UV-VIS spectroscopy, transmission electron microscope (TEM) observations. The UV-VIS spectroscopy observations were made using an OceanOptics CHEMUSB4000 UV/VIS/NIR spectrometer. The background signal of each sample was subtracted from the raw data signal by prior calibration. 
The UV-VIS data was collected and analyzed using SpectraSuite software. The TEM observations were performed using a JEOL $200 \mathrm{CX}$ at 200kv. A small drop of each sample was placed on a copper grid and allowed to dry before being examined in the transmission electron microscope. The TEM images were analyzed using the Image-Pro plus and Gatan Digital Micrograph program.

\section{Results and Discussions}

In this section we are presenting our preliminary results on Au nanoparticles ablated in neat dw, dw-chitosan, and dw-starch solutions for different ablation cases. In general, all Au nanoparticles generated here were polycrystalline particles with sizes ranging from $1 \mathrm{~nm}$ to about $30 \mathrm{~nm}$ depending on the ablation conditions. In all cases, the nanoparticles were formed initially in the plasma plume and dispersed slowly into the liquid in all directions. However, the particle dispersion was much slower in the presence of chitosan than it was in neat dw or in dw containing starch as typically shown in Figure 2. In fact, in the case with chitosan, the particles accumulated in the vicinity of the ablation region while in the case with neat $\mathrm{dw}$ or $\mathrm{dw}$ containing strach, the particles were spreading almost in all directions. This can be attributed to the difference in the liquid viscosities (viscosity of dw $+0.1 \mathrm{wt} \%$ low molecular chitosan is about $6 \mathrm{cp}$ while the viscosity of DW-0.1 wt\% medium molecular weight chitosan is $12 \mathrm{cp}$ and the viscosity of neat $\mathrm{dw}$ or $\mathrm{dw}$ containing starch is about $1 \mathrm{cp}$ ).
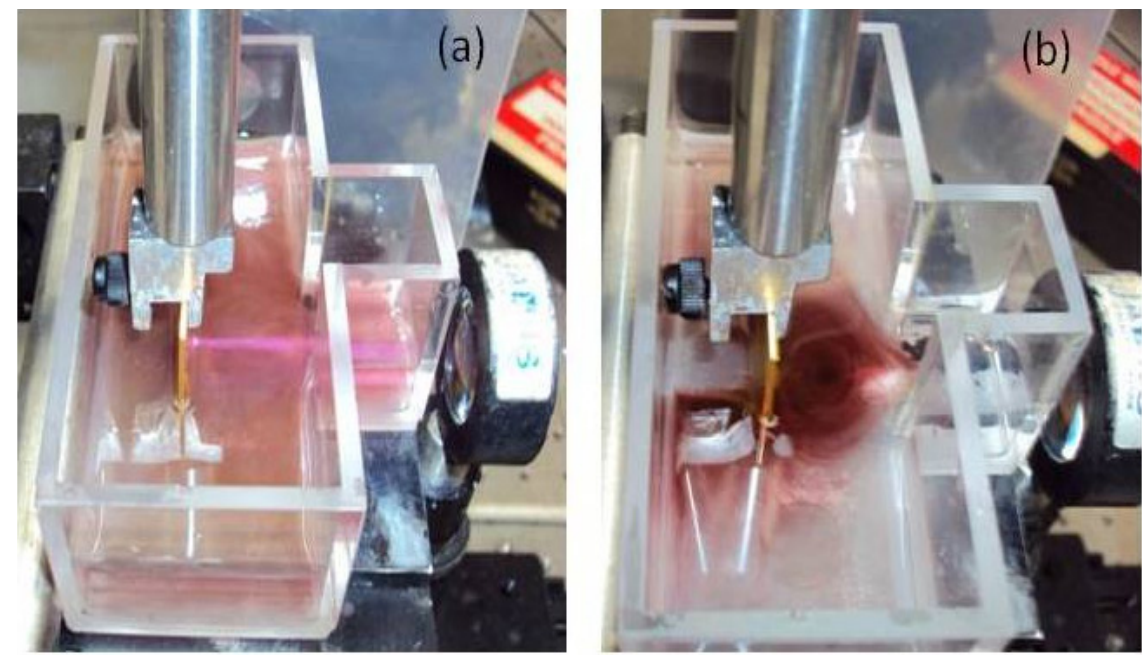

Figure 2: Effects of the liquid medium on the particle dispersion during the first 5 minutes of the ablation process: (a) in deionized water; (b) in $\mathrm{dw}+0.1 \mathrm{wt} \%$ low molecular weight chitosan.

Since particle accumulation in the focal region affects both the laser target heating and the confinement action due to the liquid in which the ablated solid is submerged, such dispersion behaviors are believed to have a significant effect on the ablation rates as well as the particle morphology. In Figure 3, we show the UV-VIS spectra obtained for Au nanoparticles ablated in dw and dw containing $0.1 \mathrm{wt} \%$ chitosan after 15 minutes. In the case that the liquid was not stirred, the ablation amount was about 4 mg and the curve representing the particles produced in $\mathrm{dw}$ had a plasmon peak located at about $525 \mathrm{~nm}$ with its shoulder extended toward the infrared region. While in dw containing $0.1 \mathrm{wt} \%$ chitosan, the ablation amounts were about $3 \mathrm{mg}$ and the plasmon peaks were slightly blue-shifted and located at about $519 \mathrm{~nm}$. When the liquid was stirred, the ablated particles were removed from the solid target quickly and the ablation rate increased significantly.
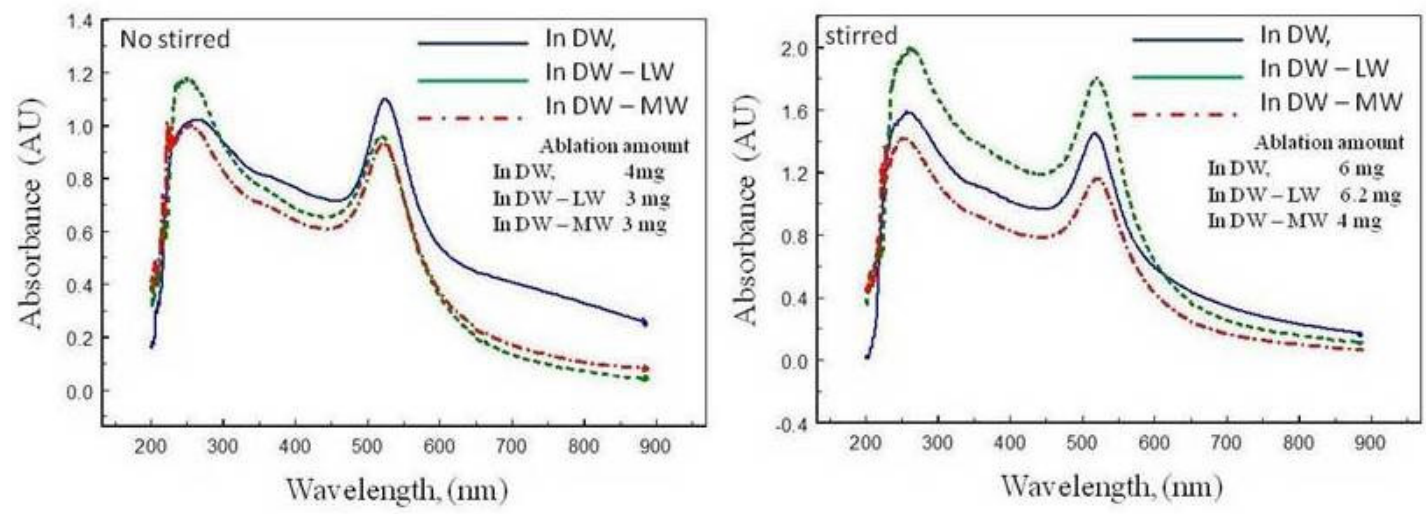

Figure 3: UV-VIS spectra of Au nanoparticles after 15 minutes of ablation in dw and dw containing 0.1 wt $\%$ chitosan. The total ablation amounts after 15 minutes of ablation were included. 
The TEM images shown in Figure 4 (a) and (b) are for the gold particles produced in deionized water using both the single and double beam ablation approaches. General observations indicated that Au nanoparticles produced in dw were big. The particle sizes from about 10 to $40 \mathrm{~nm}$ were observed when the single-beam ablation was used while the distribution was about $5 \mathrm{~nm}$ to 15 $\mathrm{nm}$ were seen when the two-beam ablation was used. These particles were not stable, they agglomerated and connected together in a random structure and settled on the cell bottom after about few hours at rest. Such the unstable behavior can be seen in Figure 5 where the luminescence of the suspension was recorded for 24 hours. To measure the luminescence from the suspension, we used a continuous beam at $532 \mathrm{~nm}$. The transmission of the excitation beam and the luminescence from the suspension were detected using a detector. Included in the figure are the photographs of the fluid vial taken at three different times. It is clear that, when the suspension was created, it was opaque to the excitation beam and the luminescence was significant in the wavelength region spreading from 600 to $900 \mathrm{~nm}$. As time went on, the intensity of the excitation beam increased while the intensity of the luminescence decreased and disappeared after about 24 hours. The corresponding fluid in the vial became clear with significant amount of black particles were seen to settle on the bottom of the vial.
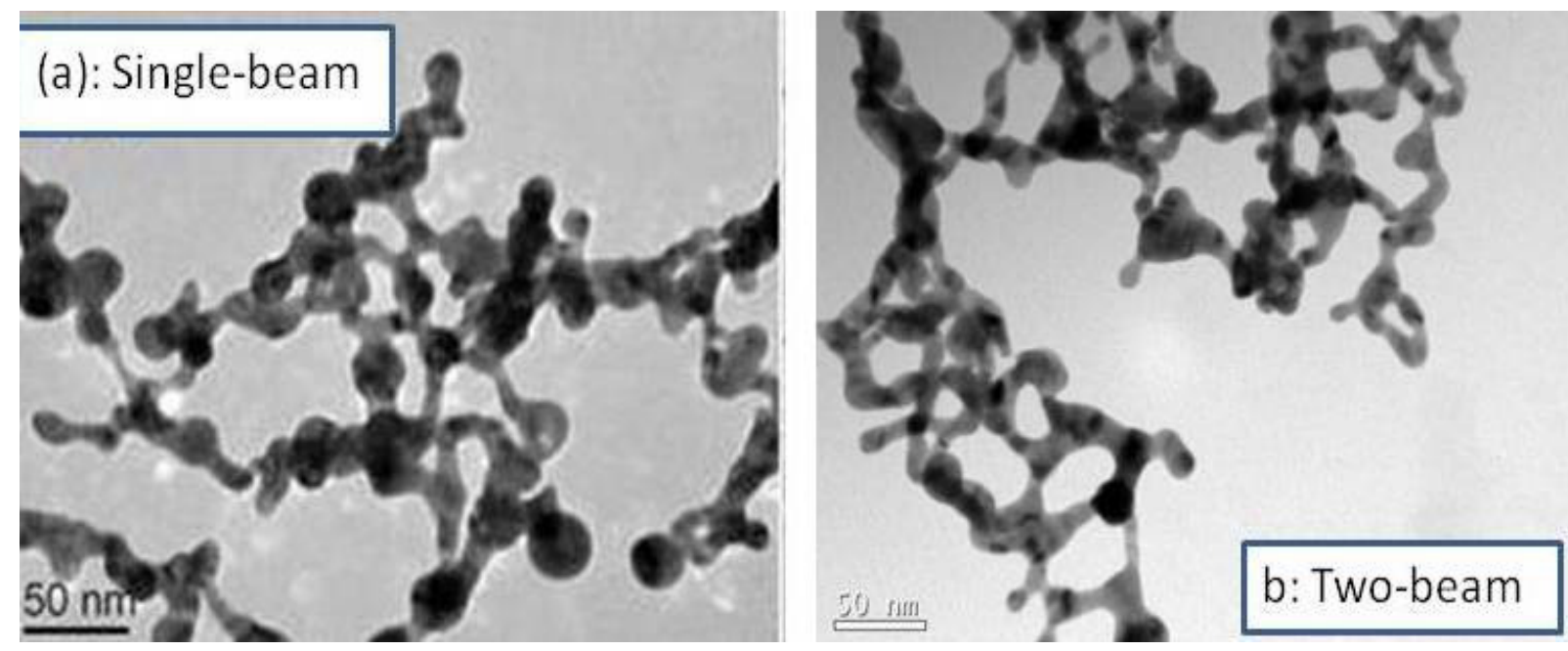

Figure 4: TEM images of Au nanoparticles ablated in dw using single and two-beam ablation approach.
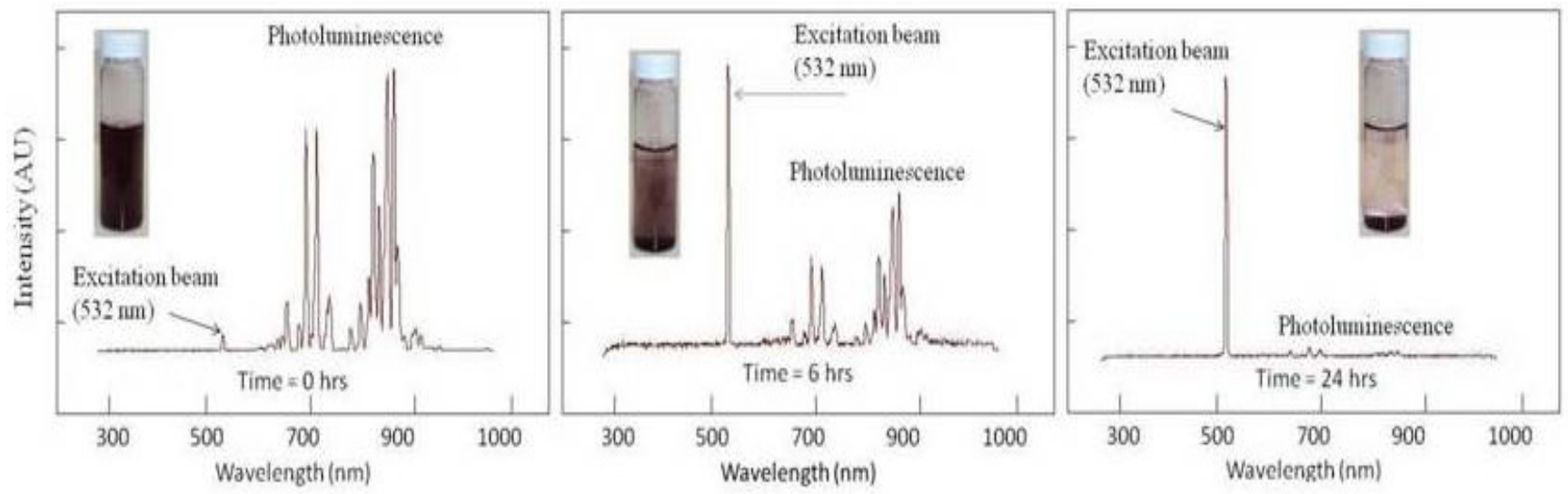

Figure 5: Luminescence from Au nanoparticles suspension ablated in neat dw, the wavelength of the excitation beam was $532 \mathrm{~nm}$.

In contrast, when gold was ablated in dw containing either chitosan or starch, the resulting suspension became stable with smaller, isolated, and well dispersed particles. Its color and luminescence remained unchanged for months as typically shown in Figure 6 for the case where the ablation was carried out in dw with chitosan as a stabilizer.

Figure 7 shows the TEM results for Au nanoparticles ablated in dw containing $0.1 \mathrm{wt} \%$ of low and medium molecular weight chitosan. It seems that the affect of the chitosan molecular weight on the particle size and shape was not well demonstrated, this might be simply because the TEM instrument does not provide high resolution images. For the single laser ablation case, the particles had a wide size distribution ranging from about from $1 \mathrm{~nm}$ to $20 \mathrm{~nm}$ in diameter. Some small particles were more isolated while others were also seen to attach to the larger one. Thus, in the presence of chitosan, the size of the ablated particles was reduced significantly but the size distribution was clearly not affected. This is similar to the results reported by Tsuji et al. [11] on silver nanoparticles. Such a size reduction observed here is due to the attachment of chitosan molecules to the surface of the ablated particles preventing them from growth and agglomeration. As a result, the particles become isolated and well dispersed and the laser beam is able to interact with more particles in the dw-chitosan solutions than in the neat water. 


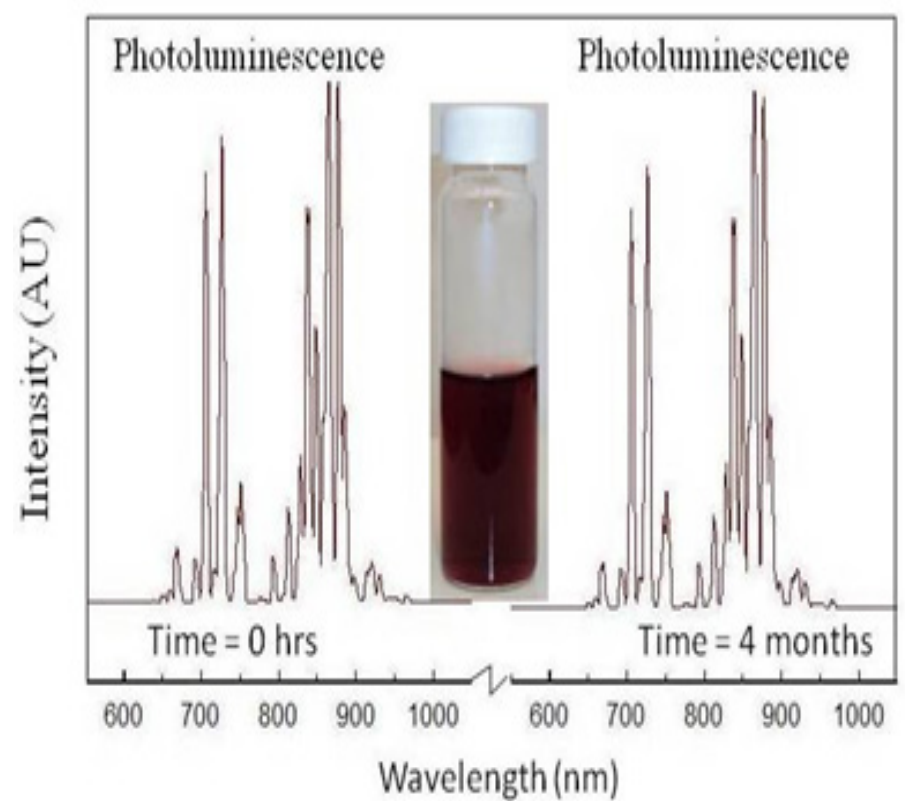

Figure 6: Luminescence from Au nanoparticles abalted in dw containing $0.1 \mathrm{wt} \%$ low molecular weight chitosan showing that the suspensions are stable.

For the two-beam ablation case, the size of the gold nanoparticles was reduced considerably, allowing the particles to be more uniformly dispersed throughout the solution. The particle sizes distribution was narrower with size ranged from $1 \mathrm{~nm}$ to $5 \mathrm{~nm}$, some particles as large as $10 \mathrm{~nm}$ were also visible. Figure 8 shows the TEM images of Au particles ablated in dw containing starch indicating that similar results on the particle size and size distributions were also obtained when starch was used in the place of chitosan. In the case of potato, however, these particles had a uniform cylindrical shape of about $20 \mathrm{~nm}$ in length and about less than $5 \mathrm{~nm}$ in diameter. Thus, Au nanorods can be synthesized by using potato starch as a stabilizer.
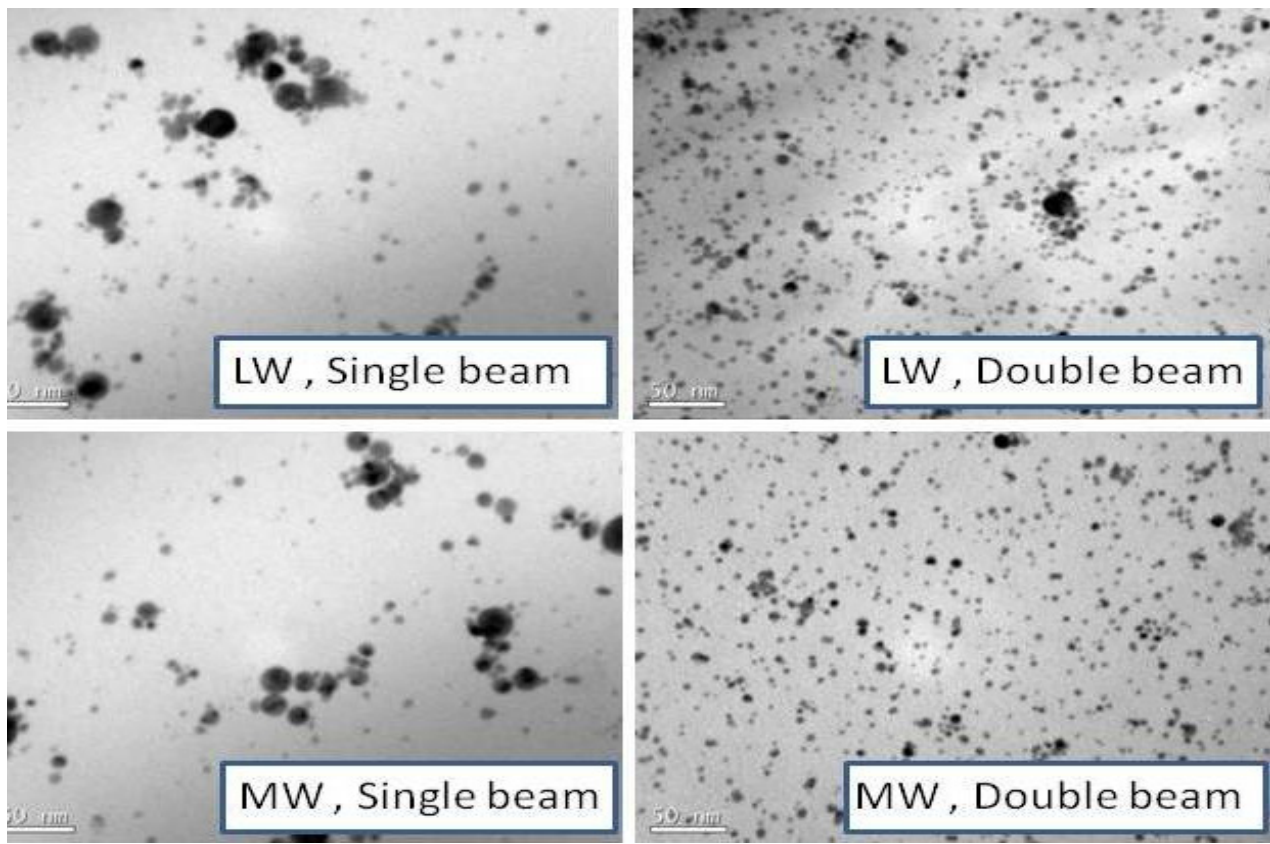

Figure 7: TEM images of Au nanoparticles ablated in dw containing $0.1 \mathrm{wt} \%$ chitosan.

Laser-induced particle fragmentation has been reported [11,12,23-26]. However, the profound reductions in the particle size and size distribution observed here cannot be attributed simply only to the coupling effects between the ablated particles and the second laser beam that can further heat, melt, and fragment the ablated particles into smaller size particles. This is because smaller particles are more reactive and they are easily agglomerated. To prevent agglomerations, the surface of these particles must be functionalized with some stabilizing molecules so that they can be isolated and stably dispersed. Tsuji et al. [11] have reported a study on the preparation of silver nanoparticles by laser ablation of a silver target in PVP solutions. 


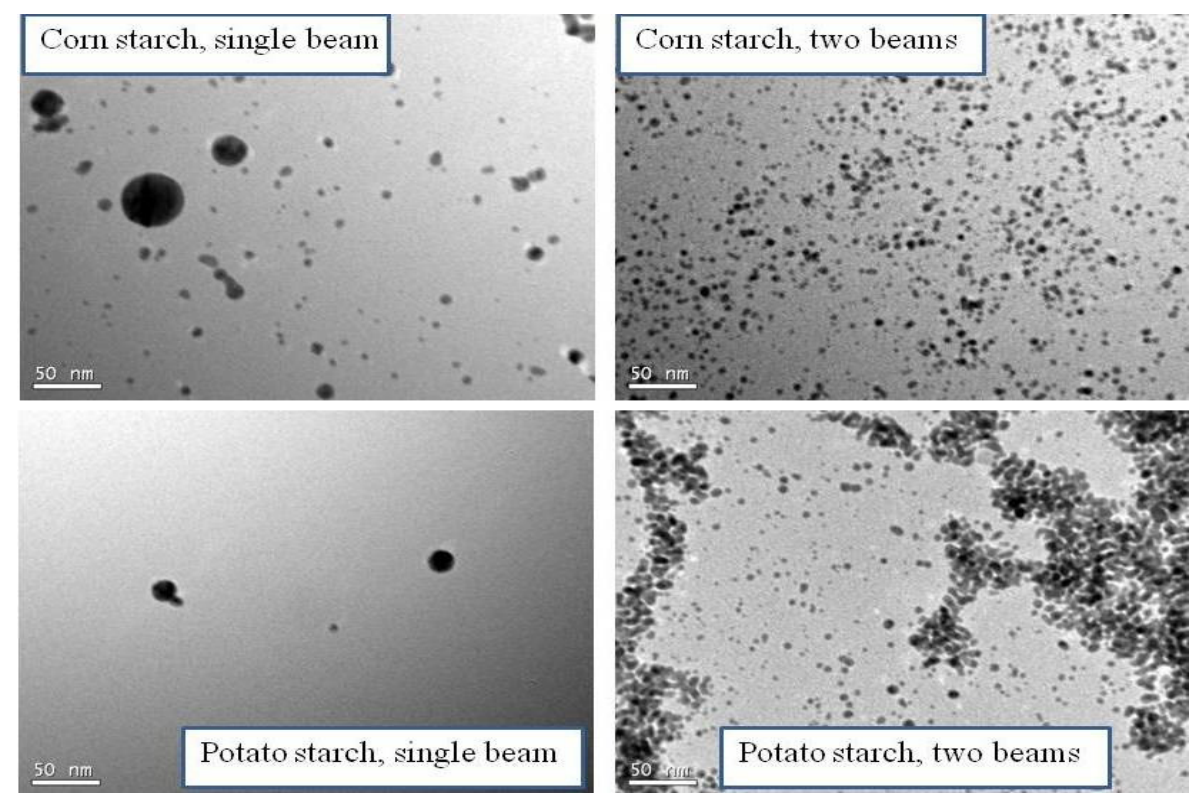

Figure 8: TEM images of Au nanoparticles ablated in dw containing $1 \mathrm{wt} \%$ starch.

Using a secondary laser irradiation onto the silver colloids prepared in neat water and in PVP solution, they reported that the population of particles smaller than $5 \mathrm{~nm}$ increased significantly in PVP solutions while in the neat water many particles larger than $30 \mathrm{~nm}$ were observed. Thus, the combined effects of chitosan or starch stabilization and laser-induced particle fragmentation are responsible for the reductions of the particle size and the size distribution shown in Figure 7 and 8.

From the results above, even with the presence of chitosan/starch, the multi-beam ablation approach produced nanoparticles with much narrower size distribution than did the single-beam ablation approach. It has been discussed by Yang [2] that the formation of nanoparticles by laser ablation in liquid involves with nucleation, phase transition, and nanocrystal growth and agglomeration processes. The nucleation time and growth time of nuclei are the two main factors that determine the ablation rate, the final size, and the size distribution of the nanoparticles produced by laser ablation in liquid. These two factors depend mainly on the particle concentration, mobility, the shock-induced particle dispersion, and the thermodynamic properties of the plasma plume which all depend strongly on the density and the viscosity of the surrounding liquid. In the presence of chitosan, the solution becomes heavier and more viscous. Particles produced in the plasma plume confined in such a heavy and viscous liquid, therefore, have more time to grow and interact with the laser beam but insufficiently with the chitosan molecules before they reach the bulk liquid. As a result, particles with different sizes are generated. In the case of multi-beam ablation, the fragmented particles are well protected by chitosan molecules. The particles sizes become smaller and the size distribution become narrower.

\section{Conclusions}

We have conducted a study on the green synthesis of nontoxic gold nanoparticles. It was found that using two-beam laser ablation in liquid together with biodegradable and biocompatible natural polymers such as chitosan or starch, stable, nontoxic, and biocompatible gold nanoparticle suspensions can be synthesized. It was also found that the two-beam laser ablation technique could produce small and well dispersed nanoparticles and yields higher ablation rates. This is mainly due to the ability of the second beam to effectively interact with the particles produced by the ablating beam.

\section{Acknowledgement}

"This report was prepared as an account of work sponsored by an agency of the United States Government. Neither the United States Government nor any agency thereof, nor any of their employees, makes any warranty, express or implied, or assumes any legal liability or responsibility for the accuracy, completeness, or usefulness of any information, apparatus, product, or process disclosed, or represents that its use would not infringe privately owned rights. Reference herein to any specific commercial product, process, or service by trade name, trademark, manufacturer, or otherwise does not necessarily constitute or imply its endorsement, recommendation, or favoring by the United States Government or any agency thereof. The views and opinions of authors expressed herein do not necessarily state or reflect those of the United States Government or any agency thereof."

\section{References}

1. Tong L, Wei Q, Wei A, Cheng JX (2009) Gold nanorods as contrast agents for biological imaging: optical properties, surface conjugation and photothermal effects. Photochem Photobiol 85: 21-32. 
2. Yang GW (2007) Laser ablation in liquids: Application in the synthesis of nanocrystals. Progress in Material Science 52: 648-98.

3. Semaltianos NG (2010) Nanoparticles by laser ablation. Critical Reviews in Solid State and Materials Sciences 35: 105-24.

4. Liu P, Cui H, Wang CX, Yang GW (2010) From nanocrystal synthesis to functional nanostructure fabrication: laser ablation in liquid. Phys Chem Chem Phys 12: $3942-52$.

5. Tarasenko NV, Butsen AV, Nevar EA, Savastenko NA (2006) Synthesis of nanosized particles during laser ablation of gold in water. Applied Surface Science 252: 4439-44.

6. Kim HJ, Bang InC, Onoe J (2009) Characteristic stability of bare Au-water nanofluids fabricated by pulsed laser ablation in liquids. Optics and Lasers in Engineering 47: 532-8.

7. Tran X. Phuoc, Soong Y, Chyu MK (2007) Synthesis of Ag-Deionized water nanofluids using multi-beam laser ablation in liquids. Optics and Lasers in Engineering 45: 1099-106.

8. Tran X. Phuoc, Howard BH, Martello DV, Soong Y, Chyu MK (2008) Synthesis of $\mathrm{Mg}(\mathrm{OH})_{2}, \mathrm{MgO}$, and Mg nanoparticles using laser ablation of magnesium in water and solvents. Optics and Lasers in Engineering 46: 829-34.

9. Hur TB, Tran X. Phuoc, Chyu MK (2009) Synthesis of Mg-Al and Zn-Al layered double hydroxide nanocrystals using laser ablation in water. Optics and Lasers in Engineering: 47: 695-700.

10. Tran X. Phuoc, Chyu MK (2009) Synthesis and rheological properties of cation-exchanged Laponite suspensions. Colloid and Surfaces A 351: 71-7.

11. Tsuji T, Thang DH, Okazaki Y, Nakanishi M, Tsuboi Y, Tsuji M (2008) Preparation of silver nanoparticles by laser ablation in polyvinylpyrrolidone solutions. Applied Surface Science 254: 5224-30.

12. Tsuji T, Okazaki Y, Higuchi T, Tsuji M (2006) Laser-induced morphology changes of silver colloids prepared by laser ablation in water - Enhancement of anisotropic shape conversions by chloride ions. Journal of Photochemistry and Photobiology A: Chemistry 183: 297-303.

13. HuangH, Yang, X (2004) Synthesis of polysaccharide-stabilized gold and silver nanoparticles: a green method. Carbohydrate Research 339: $2627-31$.

14. Shih CM, Shieh YT, Twu YK (2009) Preparation of gold nanopowders and nanoparticles using chitosan suspensions. Carbohydrate Polymers 78: 309-15.

15. Wang W, Cui H (2008) Chitosan-luminol reduced gold nanoflowers: from one-pot synthesis to morphology-dependent RPR and chemiluminescence sensing. J Phys Chem C 112: 10759-66.

16. Huang H, Yang X (2004) Synthesis of chitosan-stabilized gold nanoparticles in the absence/presence of tripolyphosphate. Biomacromolecules 5: 2340-6.

17. Besner S, Kabashi AV, Winnik FM, Meunier M (2009) Synthesis of size-tunable polymer-protected gold nanoparticles by femtosecond laser-based ablation and seed growth. J Phys Chem C 113: 9526-31.

18. Manno D, Filippo E, Giulio MD, Serra A (2008) Synthesis and characterization of starch-stabilized Ag nanostructures for sensors applications. Journal of NonCrystalline Solids 354: 5515-20.

19. Chairam S, Poolperm C, Somsook E (2009) Starch vermicelli template-assisted synthesis of size/shape-controlled nanoparticles. Carbohydrate Polymers 75 : 694-704.

20. Zhang Q, Zhang L, Li J (2007) “Green” synthesis of size controllable Prussian blue nanoparticles stabilized by soluble starch. J Nanosci Nanotechnol 7: 4557-61.

21. Vigneshwaran N, Nachane RP, Balasubramanya RH, Varadarajan PV (2007) A novel one-pot 'green' synthesis of stable silver nanoparticles using soluble starch. Carbohydr Res 341: 2012-8.

22. Raveendran P, Fu J, Wallen SL (2003) Completely "green" synthesis and stabilization of metal nanoparticles. J Am Chem Soc 125: 13940-1.

23. Kazakevich PV, Simakin AV, Shafeev GA, Viau G, Soumaré Y, et al. (2007) Laser-assisted shape selective fragmentation of nanoparticles. Applied Surface Science 253: 7831-34.

24. Tarasenko NV, Butsen AV, Nevar EA (2005) Laser-induced modification of metal nanoparticles formed by laser ablation technique in liquids. Applied Surface Science 247: 418-22.

25. Burakov VS, Tarasenko NV, Butsen AV, Rozantev VA, Neldel'ko MI (2005) Formation of nanoparticles during double-pulse laser ablation of metals in liquids. Eur Phys J Appl Phys 30: 107-12.

26. Phuoc Tran X, Soong Y, Chyu MK (2007) Synthesis of Ag-deionized water nanofluids using multi-beam laser ablation in liquids. Optics and Lasers in Engineering 45: 1099-106.

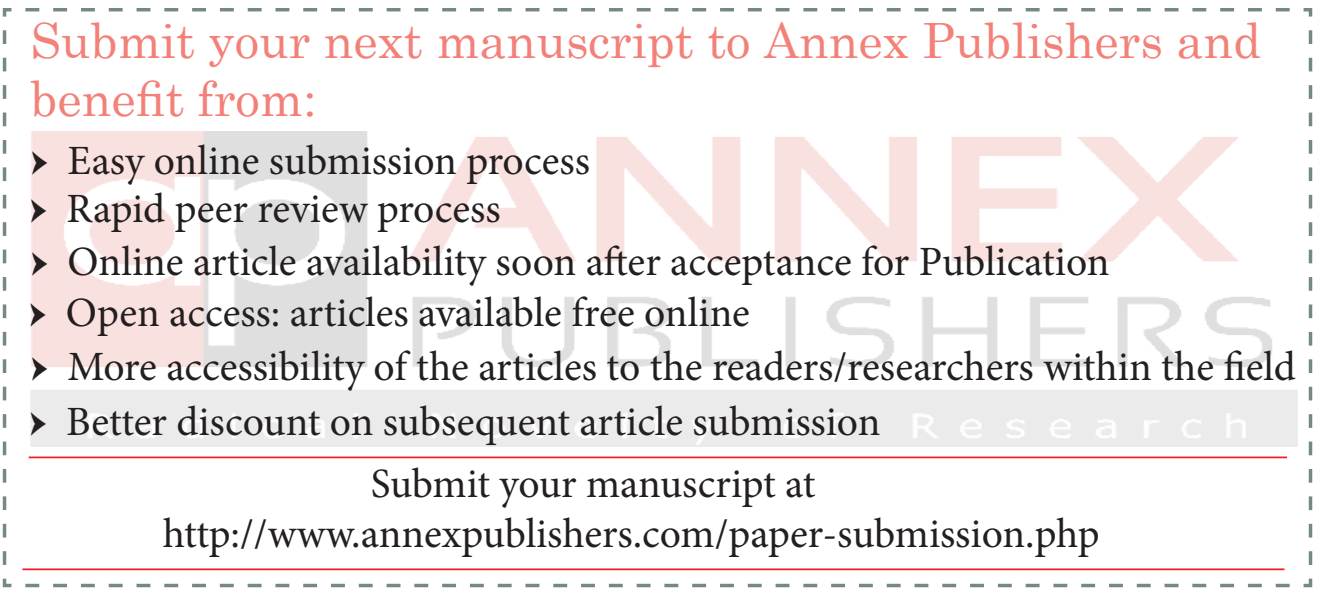

